University of New Hampshire

University of New Hampshire Scholars' Repository

Center for Coastal and Ocean Mapping

Center for Coastal and Ocean Mapping

$4-2005$

\title{
Protocols for calibrating multibeam sonar
}

\author{
Kenneth G. Foote \\ Woods Hole Oceanographic Inst. \\ Dezhang Chu \\ Woods Hole Oceanographic Inst. \\ Terence R. Hammer \\ Woods Hole Oceanographic Inst. \\ Kenneth Baldwin \\ University of New Hampshire, Durham \\ Larry A. Mayer \\ University of New Hampshire, larry.mayer@unh.edu
}

See next page for additional authors

Follow this and additional works at: https://scholars.unh.edu/ccom

Part of the Oceanography and Atmospheric Sciences and Meteorology Commons

\section{Recommended Citation}

The following article appeared in: K. G. Foote, D. Chu, T. R. Hammar, K. C. Baldwin, L. A. Mayer, L. C. Hufnagle, and J. M. Jech, 'Protocols for calibrating multibeam sonar', The Journal of the Acoustical Society of America, vol. 117, no. 4, p. 2013, 2005. http://dx.doi.org/10.1121/1.1869073. and may be found at http://scitation.aip.org/content/asa/journal/jasa/117/4/10.1121/1.1869073

This Journal Article is brought to you for free and open access by the Center for Coastal and Ocean Mapping at University of New Hampshire Scholars' Repository. It has been accepted for inclusion in Center for Coastal and Ocean Mapping by an authorized administrator of University of New Hampshire Scholars' Repository. For more information, please contact Scholarly.Communication@unh.edu. 


\section{Authors}

Kenneth G. Foote, Dezhang Chu, Terence R. Hammer, Kenneth Baldwin, Larry A. Mayer, Lawrence C. Hufnagle Jr., and J Michael Jech 


\title{
Protocols for calibrating multibeam sonar
}

\author{
Kenneth G. Foote, Dezhang Chu, and Terence R. Hammar \\ Woods Hole Oceanographic Institution, Woods Hole, Massachusetts 02543
}

Kenneth C. Baldwin and Larry A. Mayer

University of New Hampshire, Durham, New Hampshire 03824

Lawrence C. Hufnagle, Jr.

Northwest Fisheries Science Center, NOAA-NMFS, Seattle, Washington 98112-2097

J. Michael Jech

Northeast Fisheries Science Center, NOAA-NMFS, Woods Hole, Massachusetts 02543

(Received 16 July 2004; revised 13 January 2005; accepted 20 January 2005)

\begin{abstract}
Development of protocols for calibrating multibeam sonar by means of the standard-target method is documented. Particular systems used in the development work included three that provide the water-column signals, namely the SIMRAD SM2000/90- and 200-kHz sonars and RESON SeaBat 8101 sonar, with operating frequency of $240 \mathrm{kHz}$. Two facilities were instrumented specifically for the work: a sea well at the Woods Hole Oceanographic Institution and a large, indoor freshwater tank at the University of New Hampshire. Methods for measuring the transfer characteristics of each sonar, with transducers attached, are described and illustrated with measurement results. The principal results, however, are the protocols themselves. These are elaborated for positioning the target, choosing the receiver gain function, quantifying the system stability, mapping the directionality in the plane of the receiving array and in the plane normal to the central axis, measuring the directionality of individual beams, and measuring the nearfield response. General preparations for calibrating multibeam sonars and a method for measuring the receiver response electronically are outlined. Advantages of multibeam sonar calibration and outstanding problems, such as that of validation of the performance of multibeam sonars as configured for use, are mentioned. (C) 2005 Acoustical Society of America. [DOI: 10.1121/1.1869073]
\end{abstract}

PACS numbers: 43.30.Xm, 43.58.Vb, 43.30.Yj, 43.30.Pc [WMC] Pages: 2013-2027

\section{INTRODUCTION}

Multibeam sonar, with its simultaneous formation of multiple beams oriented differently in space, typically in a fan shape, ${ }^{1}$ has a long history of application to the imaging of fixed structures. Chief among these has been imaging the seafloor to determine the bottom depth over relatively broad transects or swaths. The resulting data are typically georeferenced and displayed as maps, while substantial efforts are made to avoid echoes, also called returns, from scatterers such as fish in the intervening water column.

In other applications, particularly those relating to fisheries, specialized multibeam sonars have been adapted to allow collection of returns from the water column and display of data in real time on a screen, much like conventional sonar data. ${ }^{2}$ The increased areal coverage provided by multibeam sonars without compromise of spatial resolution makes them well suited for studies of fish behavior and vessel avoidance. A number of pioneering studies have looked at the behavior of pelagic fish schools during the passage of an acoustic survey vessel including, for example, Misund and Aglen, ${ }^{3}$ using the SIMRAD SM600 sonar, with operating frequency of $34 \mathrm{kHz}$, and Gerlotto et al. ${ }^{4,5}$ and Soria et al., ${ }^{6}$ using a RESON SeaBat 6012, with operating frequency of $455 \mathrm{kHz}$. The behavior of pelagic fish schools during trawling has also been observed with the SM600. ${ }^{3}$ The swimming speed of fish has been measured using the Doppler function of the same sonar. ${ }^{7}$ Migration of schools of herring (Clupea harengus) in the North Sea has been observed, with concomitant measurements of swimming speed, by both the Simrad SR240 and SA950 sonars, with operating frequencies of 24 and $95 \mathrm{kHz}$, respectively. ${ }^{8}$ Migration behavior of schools of capelin (Mallotus villosus) in the Barents Sea has also been observed with the SR240. ${ }^{8}$ Some other applications of multibeam sonar to fish, as well as limitations in the use of the sonar, have been summarized by Reid. ${ }^{9}$

The three-dimensional nature of multibeam sonar data has also encouraged application of modern visualization techniques, allowing the complex behavior of mid-water targets to be explored interactively and in three dimensions. ${ }^{10}$ The advantage of using multibeam sonar for mid-water target identification is also being explored by the military. ${ }^{11}$

Predator-prey interactions are being studied by means of multibeam sonar. The movements of schools of herring induced by killer whales (Orcinus orca) have been measured, ${ }^{12}$ as have the interactions between juvenile herring and the Atlantic puffin (Fratercula arctica) ${ }^{13}$ In both of these studies, the RESON SeaBat 6012 sonar was used, with respective operating frequencies of 455 and $150 \mathrm{kHz}$. The behavior of Hawaiian spinner dolphins (Stenella longirostris) and pelagic prey has been studied with the new SIMRAD MS2000 sonar, with $200-\mathrm{kHz}$ operating frequency and 128 1.5-deg beams spanning a 150 -deg sector. ${ }^{14}$

The several fisheries applications mentioned here have succeeded because of the qualitative imaging capability of 
the multibeam sonar in a high-signal, low-noise environment, supplemented by registration of the echo magnitude. Calibration is generally important for imaging applications, for example, to define the limits of performance vis-à-vis the signal-to-noise ratio and to ensure consistent, distortionless performance at high signal-to-noise ratios. In other desired applications beyond those of visualization, ${ }^{10}$ a quantitative capability, hence calibration, is essential. This is true for numerical determination of concentration densities of fish and other biological organisms. It is also true for measurement of bubble populations ${ }^{15}$ and particulate concentrations near hydrothermal vents, ${ }^{16}$ for example.

There are additional advantages to calibrating multibeam sonars. For example, sonar performance can be monitored, with the ready possibility of detecting changes in this. While a multibeam sonar may be calibrated by the manufacturer prior to shipping to a customer, the owner may want to confirm its compliance with the specifications. Also, the user needs to be able to check the state of the instrument at or near to the time of application.

There are well-established procedures for calibrating sonars. A dozen different basic approaches to measuring the frequency response function of sonar receivers were listed in 1983 by Urick. ${ }^{17}$ Given the complexity of multibeam sonar, particularly the large number of beams, use of any of these approaches must be time consuming. At the same time, these calibration procedures require measurements of parts of the sonar, with the inevitable compounding of errors, and use of secondary standards, such as hydrophones, themselves requiring calibration.

Fortunately, there is an alternative procedure available for multibeam sonar, namely that of the standard target. ${ }^{18-20}$ This is widely used for calibrating scientific echo sounders at ultrasonic frequencies, and has already been used for measuring certain performance measures, including the overall system directionality, of the SIMRAD SM2000/200-kHz multibeam sonar $^{21,22}$ and $\mathrm{SM} 2000 / 90-\mathrm{kHz}$ multibeam sonar. $^{23}$

Recognizing the importance of developing simple and straightforward calibration procedures for multibeam sonar, a project has been conducted with the aim of developing convenient calibration protocols for multibeam sonars used for water-column measurement. Development of new protocols has already been described in a series of preliminary publications. $^{21,23,24}$ This development work is summarized, and the protocols are documented. It is believed that these are sufficiently detailed to serve as guides for the interested user. It is believed further that the protocols collectively will enable multibeam sonars configured for water-column measurement to be used as an integrated set of scientific echo sounders.

In the following, the test multibeam sonars and calibration targets are described, as are the seawater and freshwater facilities and associated instrumentation used in developing the calibration protocols. Methods involving acoustic measurements are reviewed, and results of the development work are summarized in the form of protocols. Some outstanding problems in multibeam sonar calibration are briefly discussed.

\section{MULTIBEAM SONARS AND TARGETS}

Essential to the development of calibration protocols for multibeam sonar was identification of appropriate sonar systems and specification of standard targets. Both subjects are addressed.

\section{A. Sonars}

Three sonar systems were selected for their routine provision of the water-column signal, which is otherwise the exception in most commercial multibeam sonar systems. In this section, details are given on the transducer array geometries. While this information is not usually regarded as being commercially confidential, it is not generally published either, not even in proprietary literature provided to owners of such sonars. The information is valuable in several ways, e.g., for use in beamforming based on individual element signals (Sec. V A) and for other processing or analytical operations, as in calculating nearfield-farfield transition characteristics (Sec. V G).

Some transducer specifications are compared in Table I. Differences in particular configurations are sufficient to require further elaboration in the text.

TABLE I. Comparison of three different multibeam sonars used in the calibration exercises.

\begin{tabular}{lccc}
\hline \hline Parameter & SM2000/90 kHz & SM2000/200 kHz & RESON Seabat 8101 \\
\hline Operating frequency (kHz) & 90 & 200 & 240 \\
Receiving array & & & 176 \\
$\quad$ No. of channels & 80 & 80 & 15.6 \\
$\quad$ Radius (cm) & 38.7 & 20.0 & 150 \\
$\quad$ Angular span (deg) & 94.8 & 88.16 & 101 \\
Displayed beams & 128 & 128 & 1.5 \\
$\quad$ No. of beams & 1.5 & 1.5 & 8 \\
$\quad$ Angular resolution (deg) & 8 & & 48 \\
Displayed-beam signals & 48 & 48 & 8 \\
$\quad$ No. of bits & & & 48 \\
$\quad$ Dynamic range (dB) & 12 & 72 & \\
Individual-channel signals & 72 & & \\
$\quad$ No. of bits & & & \\
$\quad$ Dynamic range (dB) & & & \\
\hline \hline
\end{tabular}




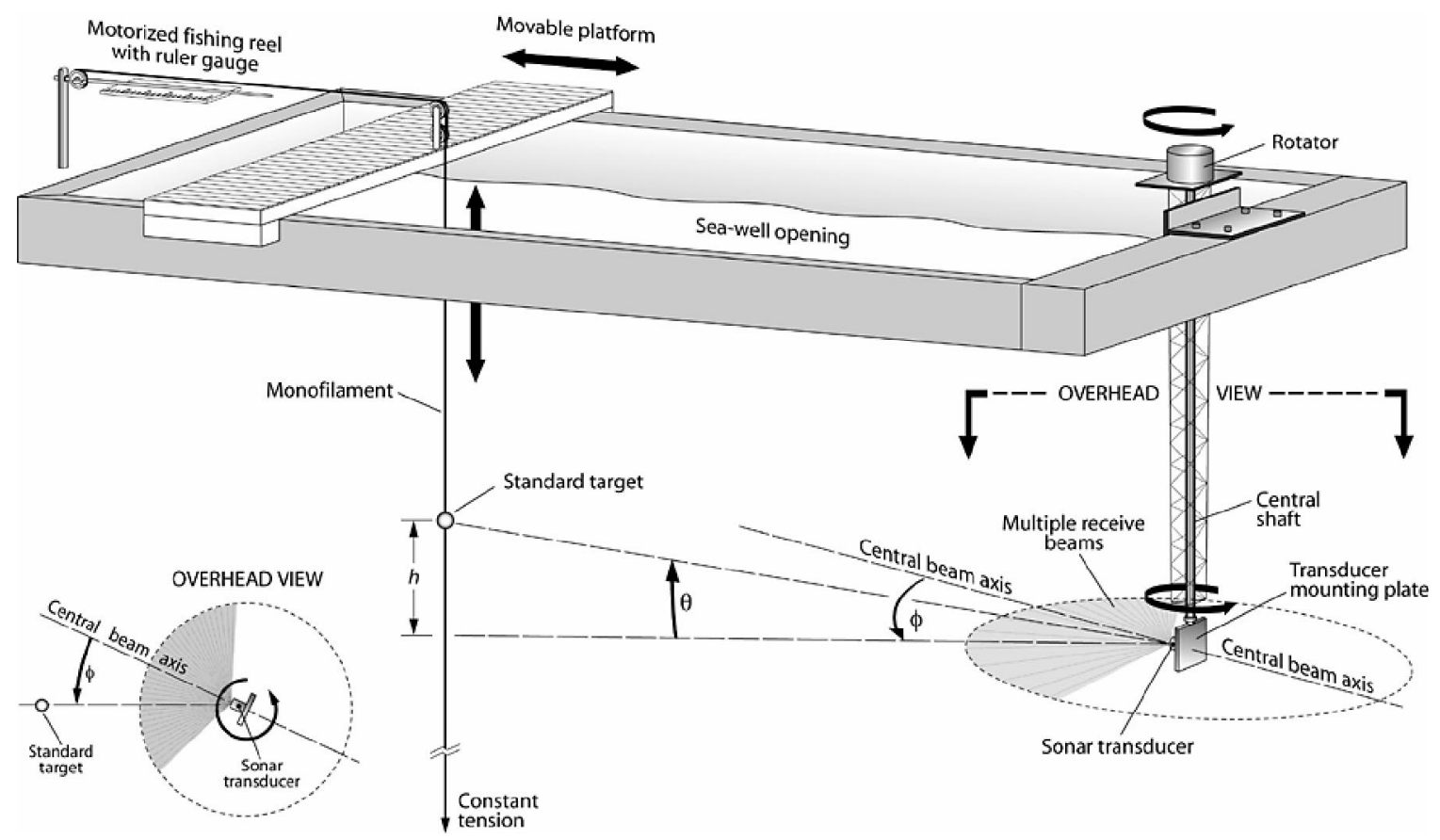

FIG. 1. Schematic diagram of the sea well as configured for calibration. For the transducer mountings, as indicated in Fig. 2, the equatorial plane is that plane running through the arc of the receiving elements, with $\theta=0$ or equivalently $h=0$, thus coinciding with the horizontal plane. The polar planes are perpendicular to the equatorial plane and contain the rotational axis of the transducer; for the particular configuration, they are vertical planes.

Transducer-mounting convention: In all cases, the transducers were mounted with their active surfaces in the vertical plane, as indicated in Fig. 1. Thus, the acoustic axis of each was horizontally oriented.

Terminology: As indicated below, the elements of each receiving array used in this study are arranged in a single row along a circular arc. The plane of this arc is called the equatorial plane. The perpendicular planes containing the rotational axis of the transducer are called the polar planes. Each particular polar plane is distinguished by its azimuthal orientation, which may define an acoustic axis if the beamforming is done through postprocessing.

\section{SIMRAD SM2000/200-kHz multibeam sonar}

The SM2000 has two separate operating modes, referred to as imaging and echo-sounding. In the imaging mode, the same transducer array is used in both signal transmission and echo reception. This array consists of 80 elements positioned with equal spacing on a circular arc (Fig. 2), thus defining the equatorial plane. Each element is rectangular, 2.54 by $19.05 \mathrm{~mm}$. Two versions are fabricated. For that of nominal span $120 \mathrm{deg}$, the radius to the outer faces of the elements is $20.00 \mathrm{~cm}$, and the interelement spacing is $1.12 \mathrm{deg}$, hence spanning a total angular sector of $88.16 \mathrm{deg}$ as measured between the centers of the end elements. For that with nominal span $150 \mathrm{deg}$, the radius to the outer faces of the elements is $10.85 \mathrm{~cm}$, and the interelement spacing is $1.13 \mathrm{deg}$, spanning a total sector of $155 \mathrm{deg}$. In the associated digital signal processor, a total of 128 receive beams are formed, each with beamwidth $1.5 \times 20$ deg in the respective horizontal and vertical planes.

In the echo-sounding mode, the described transducer array is used only in reception. Transmission is effected by an

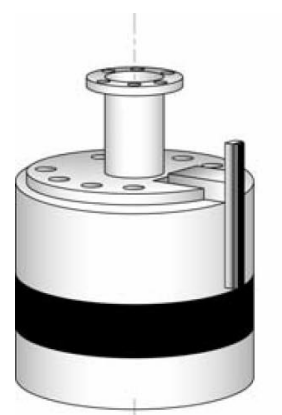

SeaBat $8101 / 240 \mathrm{kHz}$

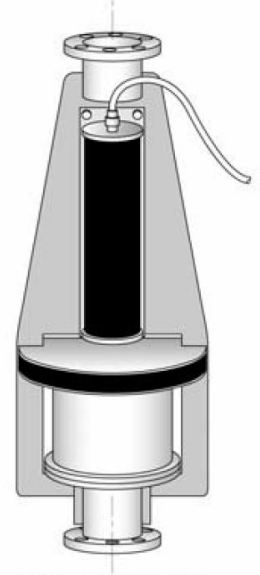

SM $2000 / 200 \mathrm{kHz}$
SM $2000 / 90 \mathrm{kHz}$

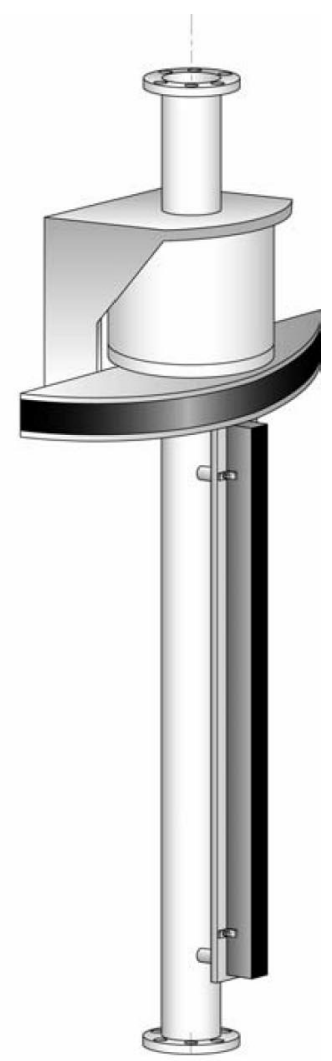

FIG. 2. Schematic diagrams of transducer-mounting plates for the SeaBat 8101 240-kHz multibeam sonar, SM2000/200-kHz multibeam sonar, and SM2000/90-kHz multibeam sonar. The black rectangular areas designate the protective coverings of the external transmitter. The black curved areas designate the same for the receiving arrays. In the case of the SM2000 models in the imaging mode, the external transmitter is not used, and the curved arrays are used both for transmission and reception. In the case of the SM2000 models in the echo-sounding mode, the external transmitter is used. 
external line array of 50 elements of total length $27.6 \mathrm{~cm}$ oriented along the vertical, thus in the central polar plane perpendicular to the equatorial plane of the receiving array (Fig. 2), and thence forming a Mills cross. ${ }^{17}$ Each transmitting element is a narrow band of width $2.1 \mathrm{~mm}$ spanning 177 deg of a circular arc of radius $44.45 \mathrm{~mm}$. The entire array appears to be a half-cylinder. The elements are stacked with a center-to-center distance of $5.6 \mathrm{~mm}$. They are weighted in amplitude, i.e., amplitude shaded. ${ }^{17}$ The computed nearfield distance is $11 \mathrm{~m} .^{25}$ Through digital signal processing, 128 receive beams are formed for the display, with nominal beamwidths $1.5 \times 1.5 \mathrm{deg}$. The number of beams that can be formed on an external processor is arbitrary.

The distinction between the two modes is recognized by a particular naming convention, which is adhered to in this work. For the general system, with optional use of either mode, reference is made to the SM2000 multibeam sonar. For the particular configuration in which the external transmitter is used, reference is made to the SM2000 multibeam echo sounder.

The transmitted signal in both modes is typically a finite, or pulsed, sinusoid at $200 \mathrm{kHz}$. The duration may be specified directly by the user or indirectly based on the specified range. For target ranges of order 5-25 m, which are typical in standard-target calibrations of high-frequency sonars, a pulse duration of $100 \mu$ s was generally used. The pulse repetition frequency was determined automatically in accordance with the range, typically about $1 \mathrm{~Hz}$ or $1 \mathrm{ping} / \mathrm{s}$. Notwithstanding the appearance of user control over the settings, the sonar itself is configured internally with certain constraints that cannot be exceeded. In addition, the sonar can neither accept nor generate an external trigger signal, which may be regarded as highly inconvenient in many applications.

The sonar displays echoes as received on 128 beams over the total angular sector of 120 or $150 \mathrm{deg}$. Significantly, the sonar can record either the raw complex echo signals from each of the 80 elements or the 128 beamformed signals. Beamforming performed within the sonar digital signal processor (DSP) uses a Hamming-type amplitude-weighting. ${ }^{26}$ Beamforming performed through postprocessing external to the sonar DSP uses Chebyshev-type amplitude-weighting. ${ }^{17}$ Other shading schemes may be implemented when using the individual element outputs, and other apertures may also be defined.

The data are digitized with 8-bit resolution for display and with 12-bit resolution for recording purposes. The nominal dynamic range of the recorded data is thus $72 \mathrm{~dB}$.

\section{SIMRAD SM2000/90-kHz multibeam sonar}

The architecture of this sonar, which operates at $90 \mathrm{kHz}$, is similar to that of the $200-\mathrm{kHz}$ sonar described above. Similarly to the $200-\mathrm{kHz}$ sonar, the $90-\mathrm{kHz}$ sonar operates in both imaging and echo-sounding modes. The collocated imaging array (Fig. 2) also consists of 80 identical elements spaced at intervals of $1.20 \mathrm{deg}$ along a circular arc of radius $38.74 \mathrm{~cm}$ measured to the outer face of the element and spanning 94.8 deg as measured between the centers of the end elements. The dimensions of the individual rectangular elements are $5.08 \times 64.8 \mathrm{~mm}$.

The external transmitter is a linear array $86.6 \mathrm{~cm}$ in length oriented perpendicularly to the equatorial plane of the receiving array (Fig. 2). This consists of 12 pairs of elements. Each element is rectangular with dimensions 3.8 $\times 64.8 \mathrm{~mm}$. The center-to-center distance between elements in a pair is $17.8 \mathrm{~mm}$. The center-to-center distance between pairs, aligned end-on, is $65.3 \mathrm{~mm}$.

The digital signal processor forms 128 beams for display. The nominal beamwidths are $1.5 \times 20 \mathrm{deg}$ in the imaging mode, without use of the external transmitter, and 1.5 $\times 1.5 \mathrm{deg}$ in the echo-sounding mode, with use of the external transmitter.

Transmit signals are pulsed sinusoids with a center frequency of $90 \mathrm{kHz}$. For nominal target ranges of order 5-25 $\mathrm{m}$, which are used in standard-target calibrations, a pulse duration of $300 \mu \mathrm{s}$ was typically selected.

As with the $200-\mathrm{kHz}$ sonar, the $90-\mathrm{kHz}$ sonar provides both the beamformed signals and individual element signals, but only one at a time depending on operator choice. Thus, the user can choose to perform the beamforming independently of the sonar processor.

As with the $200-\mathrm{kHz}$ multibeam sonar, the digitized data are displayed with 8-bit resolution, but recorded with 12-bit resolution. Thus, the nominal dynamic range of the recorded data is $72 \mathrm{~dB}$.

\section{RESON SeaBat 8101 multibeam sonar at $240 \mathrm{kHz}$}

This sonar consists of separate transmitting and receiving transducer arrays (Fig. 2). The transmitting array is a linear array of 36 elements conforming to a slender cylinder of radius $2.5 \mathrm{~mm}$, each $5.28 \mathrm{~mm}$ in length and separated by a gap of $0.7 \mathrm{~mm}$, hence with total length $21.46 \mathrm{~cm}$. The transmitting array is oriented perpendicularly to the equatorial plane of the receiving array. A swath exceeding $150 \mathrm{deg}$ is insonified. Shading is employed to reduce the on-axis transmit signal strength, as this typically corresponds to the nadir in bathymetric mapping exercises. The same shading achieves a one-way, received sidelobe level of about -20 $\mathrm{dB}$, as computed.

The receiving array is composed of 176 elements evenly spaced on a circular arc of radius $15.6 \mathrm{~cm}$. Each receiving element is rectangular, with height $16.88 \mathrm{~mm}$ and width 3.1 $\mathrm{mm}$. The center-to-center distance subtends an arc of 1.5 deg. The system forms $1011.5 \times 1.5$-deg beams over an angular sector of $150 \mathrm{deg}$.

In contrast to the SM2000, the SeaBat 8101 operates in only one mode, with separate transmitting and receiving arrays. Thus, it functions like the SM2000 multibeam echo sounder, but is referred to in this work as a multibeam sonar, consistent with the manufacturer's own naming convention.

Although the SeaBat 8101 does not support externaltrigger operation, it does accept a synchronized clock signal from another computer. The computer that transmits the synchronization signal can also accept motor-position information from the motor controller. The beamformed data are 
digitized and stored with 8-bit resolution. The nominal dynamic range is thus $48 \mathrm{~dB}$.

\section{B. Standard targets}

A number of standard targets were selected or devised. In every case these were based on the spherical form. Principles of optimization of the diameter for both narrow-band and broadband sonars ${ }^{18,27-29}$ were not applied in the present work. Rather, for reasons of convenience, a number of suboptimal targets were chosen based on ready availability. These included spheres made of electrolytic-grade copper with diameters 23 and $60 \mathrm{~mm}$, tungsten carbide with $6 \%$ cobalt binder with diameters 20 and $38.1 \mathrm{~mm}$, aluminum with diameter $60 \mathrm{~mm}$, and titanium with diameter $47 \mathrm{~mm}$.

To achieve substantially higher target strengths, several focusing spheres ${ }^{30-33}$ were fabricated. ${ }^{34}$ Two of these were based on spherical shells made of high-silicon bronze with external diameters $101.6 \mathrm{~mm}$ and shell thickness $3 \mathrm{~mm}$. One was outfitted with a movable piston plug, diameter 14.22 $\mathrm{mm}$, and the other with a simple shallow screw. Another focusing sphere was fabricated from a hollow rubber ball of diameter $88.9 \mathrm{~mm}$, with thickness varying from 1.7 to 2.1 $\mathrm{mm}$ (average $1.9 \mathrm{~mm}$ ). The three hollow spheres were vacuum-filled with the fluorocarbon liquid FS-5, also known by the tradename Fluorlube. ${ }^{35}$

In some cases, the target strengths of the several spheres were either computed according to the respective theories for solid elastic spheres, ${ }^{36,37}$ noting corrections, ${ }^{18}$ or the appropriate limiting case as given by Goodman and Stern, ${ }^{38}$ or for spherical shells, ${ }^{38}$ assuming accepted values for the material properties. In other cases, the target strengths were determined according to comparative measurements. The results are shown in Table II.

\section{CALIBRATION FACILITIES AND THEIR INSTRUMENTATION}

Both seawater and freshwater facilities were instrumented for the work of developing calibration protocols. Earlier preliminary reports ${ }^{34,39,40}$ are summarized and updated.

\section{A. Sea well}

Iselin Dock at the Woods Hole Oceanographic Institution is a large dock supported by concrete pilings. Large reinforced concrete blocks have been removed from a particular area, exposing a sea well with rectangular opening 6 $\times 13 \mathrm{~m}^{2}$ (Fig. 1). The hard bottom beneath this opening slopes from about 15 to $20 \mathrm{~m}$. Another area approximately 22-24 $\mathrm{m}$ from the transducer mounting apparatus is also easily accessible for mounting equipment such as calibration targets.

The sea well is within about $700 \mathrm{~m}$ of the Woods Hole passage. Because of its high-tidal-current velocities, ${ }^{41}$ stratification is at most weak. Because of the performance of seawell calibrations over quite short ranges, effects of such stratification are negligible.

Interestingly, the tidal current at Iselin Dock is rectified. The flood occurs with substantial current towards the southeast, typically of the order of 1.5 knots. During the ebb, however, the water level falls or settles with very slight and variable current. The tidal range is typically of order $0.8 \mathrm{~m}$. It has been possible to perform measurements during both tidal states, with those made during the flood being less variable.

The sea well was equipped with a variety of instruments and gear for deploying and rotating sonar transducers and for suspending and controlling targets at known positions in the transducer beam. Because of the wide range of uses of the sea well, all mountings were made in a provisional manner, but with drilled holes or markings for future reassembly of the facility.

A standard, 6-m-long shipping container was provided or configured as a laboratory for the electronic instruments and computers during each calibration trial. Mains power of both 110 and $220 \mathrm{~V}$ at $60 \mathrm{~Hz}$ was supplied by cable. The laboratory was placed immediately beside the sea well for operational convenience, specifically to minimize the cable distance to the transducers and rotation apparatus and to facilitate communication among the various participants.

\section{Transducer-mounting system}

During calibration trials, a 6-m-long steel-trussed tapered antenna tower weighing $150 \mathrm{~kg}$ was affixed to a mas-

TABLE II. Nominal target strength (TS) values of standard targets as computed and as inferred at $200 \mathrm{kHz}$ by means of the SM2000 multibeam echo sounder. The TS values shown in the parentheses have been computed at precisely $200 \mathrm{kHz}$ according to the single-frequency theory ${ }^{36}$ based on tabulated values for the physical properties of the respective target and applicable conditions of temperature and salinity. The inferred TS values have been calculated relative to the average target strength of the 38.1-mm tungsten carbide sphere (WC38.1).

\begin{tabular}{lcccc}
\hline \hline Target & $\begin{array}{c}\text { Diameter } \\
(\mathrm{mm})\end{array}$ & $\begin{array}{c}\text { Relative TS } \\
(\mathrm{dB})\end{array}$ & $\begin{array}{c}\text { Inferred TS } \\
(\mathrm{dB})\end{array}$ & $\begin{array}{c}\text { Computed TS } \\
(\mathrm{dB})\end{array}$ \\
\hline AL60 & 60 & $96.52 \pm 1.83$ & -35.31 & -34.46 \\
& & $96.41 \pm 1.02$ & -35.42 & -34.46 \\
WC38.1 & 38.1 & $92.43 \pm 0.76$ & -39.38 & -39.36 \\
& & $92.47 \pm 0.87$ & -39.34 & -39.36 \\
WC20 & 20.0 & $88.99 \pm 0.23$ & -42.84 & -44.99 \\
High-silicon bronze & 25.4 & $94.87 \pm 0.17$ & -36.96 & -35.09 \\
Focusing sphere 1 & 101.6 & $96.74 \pm 0.95$ & -38.36 & -38.43 \\
Focusing sphere 2 & 88.9 & $93.47 \pm 1.28$ & -46.95 & \\
Stainless steel & 50.8 & $93.40 \pm 1.47$ & $84.88 \pm 0.60$ & \\
Titanium & 47 & & & \\
\hline \hline
\end{tabular}


sive I-beam spanning the sea-well opening at the northwest end. The tower was held securely in place by a mechanical lock under gravity. The tower was strapped to the lower part of the I-beam mounting plate to resist the bending moment caused by the flooding tide when acting on the tower and transducer submerged at nominal 3-m depth.

An aluminum pipe is centrally mounted in the tower to serve as a shaft for holding and rotating the transducer. The shaft is guided by holes in the steel endplates and an intermediate plate added to reduce flexure. Low-friction bearings are mounted at each hole.

The shaft is held by the rotation apparatus, described in the next section. A flange with six bolts is mounted on the lower end to serve as a universal coupling joint for the transducer plate.

Given significant differences in the shape and size of each of the multibeam sonar transducers, a separate mounting plate was fabricated in aluminum to hold the respective transducer in a secure manner. Longer mounting plates for holding separate transmitting and receiving arrays were generally braced with aluminum angle-brackets for stiffness under flooding conditions. Examples of mounting plates with attached transducers are shown in Fig. 2.

In the case of separate transmitting and receiving transducer arrays, alignment of the several beams could be effected by shimming one of the arrays relative to the other. This was done for the SM2000/90-kHz multibeam echo sounder to reduce effects of parallax given the relatively short range of the target.

\section{Rotation apparatus}

A commercial antenna motor made by M2-Antenna Systems, the MT1000 antenna azimuth positioner, was adapted to serve as a precision rotator. The apparatus was mounted atop the central shaft of the tower, supporting both thrust and radial loads.

In the course of adapting the rotator, the central gear was replaced by one made of marine bronze. The original dcmotor was also replaced with an ac-motor with 14-bit absolute encoder and counter. The rotator can turn about a 360deg arc, thus achieving a nominal precision of $\pm 0.01 \mathrm{deg}$, well within the design goal of $\pm 0.05 \mathrm{deg}$.

The accuracy of the rotator was established by measuring the deflection of the beam of a laser pointer mounted atop the central transducer-rotation shaft. Under commanded rotations, the position of the center of the red laser spot on the bridge platform, for example, was determined to within $\pm 5 \mathrm{~mm}$ at a nominal range of $11.7 \mathrm{~m}$. This was found to be repeatable over a period of days, hence with demonstrable accuracy to within $\pm 0.025 \mathrm{deg}$.

The alignment of the transducer at the end of the $6.5-\mathrm{m}$ shaft with the rotator was confirmed by attaching an outrigger to the rotator and dropping a plumb line from the outrigger to a corresponding outrigger affixed to the shaft immediately above the transducer plate, while the transducer tower was held vertically by a mobile crane. The distance from the shaft centerline to the plumb bob centerline was $47.1 \mathrm{~cm}$. Very small adjustments could be made to the alignment by exploiting the small degree of play in the flange coupling. It is estimated that the transducer could be aligned to within $\pm 0.5 \mathrm{~mm}$ over $4 \mathrm{~m}$, or to within $\pm 0.007 \mathrm{deg}$.

Rotator positions were sent in ASCII format via serial link, RS232, to the SM2000 and were recorded with the SM2000 raw data files. There was a five-ping delay between the actual rotator position and the ping that recorded the position.

\section{Target-suspension and-positioning systems}

A number of systems were procured or assembled for suspending standard targets and controlling their positions in the transducer beam. One system was adapted from that currently used on board fisheries research vessels. ${ }^{19,20}$ The target was held by each of three monofilament nylon lines connected to motorized fishing reels, with outriggers mounted on barrier timbers, two on one side of the sea well and one on the opposite side. The position of the target was controlled by means of joysticks from the electronics laboratory.

In a second system, indicated schematically in Fig. 1, the target was suspended by a central monofilament line spooled on a motorized fishing reel. A second monofilament nylon line was attached to the central line and threaded through a block resting on the bottom of the sea well directly below the target to a second block. This was attached to a safety beam bordering the well, with lead weight suspended at the submerged end of the line to maintain constant tension, whatever the tidal state.

A third system was derived from the second by attaching four additional lines to the target. ${ }^{34}$ Each of these was tensioned by running the respective line through a block to an attached weight. Each block was supported by the sea well bulkhead mounted along the long sides. The four blocks were arranged at the corners of a square. This additional tensioning was obliquely upwards, offering stability against lateral, tidally induced currents.

The second system was most used in the trials. The vertical suspension line was run through a block mounted on a platform bridging the sea well to a motorized fishing reel (Fig. 1). A variable voltage motor was used to control the fishing reel. The line was run directly over a meter ruler, allowing depth adjustments to within $\pm 0.5 \mathrm{~mm}$. At a range of $11.7 \mathrm{~m}$, this corresponds to an angular uncertainty of $\pm 0.003 \mathrm{deg}$.

\section{B. Freshwater tank facility}

The Chase Ocean Engineering Laboratory at the University of New Hampshire (UNH) has a freshwater tank that is nominally 12 by $18 \mathrm{~m}$ in plan view, with 6-m water depth. Modifications were made to this tank to facilitate the required acoustic measurements. These modifications fundamentally concerned the placement and control of the transducer under test in the tank. There were three fundamental issues to consider in achieving this goal: $X-Y-Z$ transducerpositioning, rotation, and system integration.

\section{1. $X-Y-Z$ transducer-positioning system}

The $X-Y$ positioning in the plan view of the tank was accomplished through the addition of an industrial crane-rail system spanning the 12-m width of the tank, and moving 


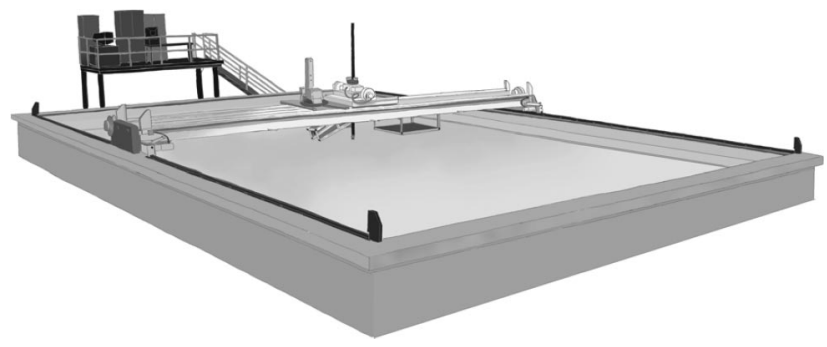

FIG. 3. Schematic diagram of the freshwater tank as configured for calibration.

along the longer, 18-m dimension (Fig. 3). The span accommodated a carriage, which moved across the 12-m span and was designed to secure the rotator. The rotator was mounted on the carriage and held the transducer under test, while controlling the incremental rotation about the vertical or $Z$ axis. The rotator held a carbon fiber pole to which the transducer under test was attached (Fig. 4). The $Z$ depth of the transducer was adjusted by securing the pole in the three-jaw chuck at the appropriate location.

\section{Rotator}

Rotation was accomplished using a Yuasa International, three-jaw chuck rotator (SUDX-320) with a programmable controller (UNDC-100). This rotator was selected due to the zero-backlash claim, and the ability to effect small increments of angular rotation, $0.1 \mathrm{deg}$. The rotator was readily mounted on the carriage. The transducer-mounting pole and rotation shaft was placed in the three-jaw chuck. A universal transducer-mounting flange was attached to the bottom of the pole. The pole was fabricated from carbon fiber, and the length was adjusted by adding sections outfitted with locally fabricated centering couplings.

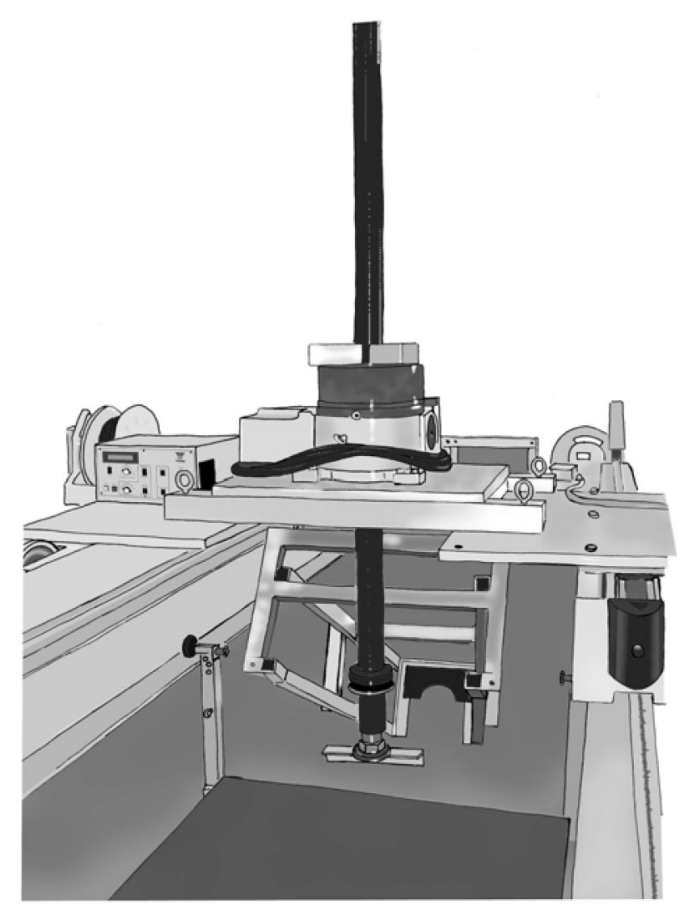

FIG. 4. Configuration of the transducer and its mounting system in the freshwater tank.
The rotator performance was verified by means of a machinist dial indicator to measure the arc length traveled due to an incremental angular input. Two-degree arcs were randomly selected around the circumference. Each 2-deg arc was traversed with $0.1-\mathrm{deg}$ increments in both directions. Precision control over the angle and the ability of the rotator to return to the place where it began, to within the accuracy limit of $\pm 0.1 \mathrm{deg}$, were both demonstrated. Details of this system development are reported in Baldwin et al. ${ }^{40}$

\section{System integration}

System integration was achieved using LabView@ software. An interpreter program was acquired which enabled LabView to communicate with the rotator controller. Subsequently other code was developed to facilitate the tasks required to perform a beam pattern measurement. Transmit signal generation, receive signal acquisition, transmit-receiverotation coordination, and beam-pattern plotting were the basic functions. The specific linking of these functions was an artifact of the particular source-receiver-target or hydrophone configuration.

\section{Target suspension and positioning}

Target suspension and positioning were effected with a single line of monofilament nylon deployed through a block at the end of an outrigger. The line was attached to a motorized fishing reel, allowing fine control of the target depth, as described in more detail above in Sec. III A 3.

\section{METHODS}

A number of procedures preparatory to calibrating sonars have already been described in the previous section. These include alignment of the transducer and rotator and possible shimming of separate transmitting and receiving arrays.

Another general preparatory measure is that of measuring the hydrographic state of the calibration environment. Vertical temperature and salinity profiles should be taken at intervals. Additionally, time series of temperature and salinity might be recorded at the transducer depth. During the particular calibration trials in the sea well, vertical conductivity-temperature-depth (CTD) profiles were taken, indicating that the seawater was well mixed. At other times, the CTD sensor was placed at the depth of the transducer to collect time series of temperature and salinity. The average temperature, salinity, and sound speed measured throughout one trial were $2.88 \pm 0.02{ }^{\circ} \mathrm{C}, 31.60 \pm 0.25 \mathrm{PSU}$, and 1457.5 $\pm 0.4 \mathrm{~m} / \mathrm{s}$, respectively.

Methods involving acoustic measurements are described in this section. Some of these are common to calibration of both scientific echo sounders and multibeam sonars; they are included here for the sake of completeness.

Implicit in the measurements for the SM2000 multibeam echo sounders are real-time storage and rapid retrieval of the echo signals from the individual receiving transducer elements and availability of operational software for beamform- 
ing. Algorithms for beamforming have been described in some detail for multibeam sonar. ${ }^{21,22}$ These follow the general method. ${ }^{17,42}$

\section{A. Target positioning and mapping central polar beam pattern}

A standard target can be placed at an essentially arbitrary position in the sonar beam by purely geometrical considerations. Not all positions are equally favorable, it must be emphasized. It is generally desirable to avoid reverberation from surfaces and possible extraneous scatterers in the water column, e.g., fish and underwater moorings. It is also desirable, if not absolutely necessary, to make measurements in the transducer farfield.

In practice it is easier to perform relative positioning of the target in the transducer beam by placing the target at the approximate depth of the centered transducer, then finding the coincident horizontal and equatorial planes of maximum sensitivity by moving the standard target systematically through a series of depths and making multiple measurements at each target depth. Beamforming is then performed, and the resulting data are fit in a least-squares sense by a quadratic or other nonlinear function. The beamformed data describe the central polar beam pattern.

Examples of resulting data derived from measurements with the SM2000/90-kHz multibeam echo sounder are shown in Fig. 5. In the first example, taken from the sea-well facility, a standard target at 23-m range was lowered, and then raised, systematically at constant increments of $10 \mathrm{~cm}$ or $0.25 \mathrm{deg}$. In the second example, taken from the freshwater-tank facility, the same standard target at $11.7-\mathrm{m}$ range was used and the procedure was repeated, but at $2-\mathrm{cm}$ increments, hence with nominal resolution of $0.10 \mathrm{deg}$.

\section{B. Measurement of system response and choosing receiver gain function}

The performance of a system is considered to be linear if a change in the amplitude of an input signal results in a linearly proportional change in the amplitude of the output signal. While there is no need for a system to perform linearly for it to be used quantitatively, given that it performs monotonically, the system response needs to be determined. Among other reasons, the sonar will be most useful if its gain function enables both weak and strong echo signals from interesting targets to be registered without loss due to inadequate sensitivity or saturation, with clipping, due to inadequate dynamic range. The general effect of clipping may be hard, with abrupt change in any amplitude exceeding a threshold value to the threshold value itself, or soft, with gradual approach to the threshold value, where the exact relationship is nonlinear. The general case has been treated under the name of band-pass limiting. ${ }^{43}$ The importance of choosing the gain function is evident from practical considerations of dynamic range: the test multibeam sonars used in this study have rather limited dynamic ranges, especially relative to those of modern scientific echo sounders. ${ }^{44}$

Multibeam sonars generally have a number of available transmit-power settings. In keeping with the aim of develop-
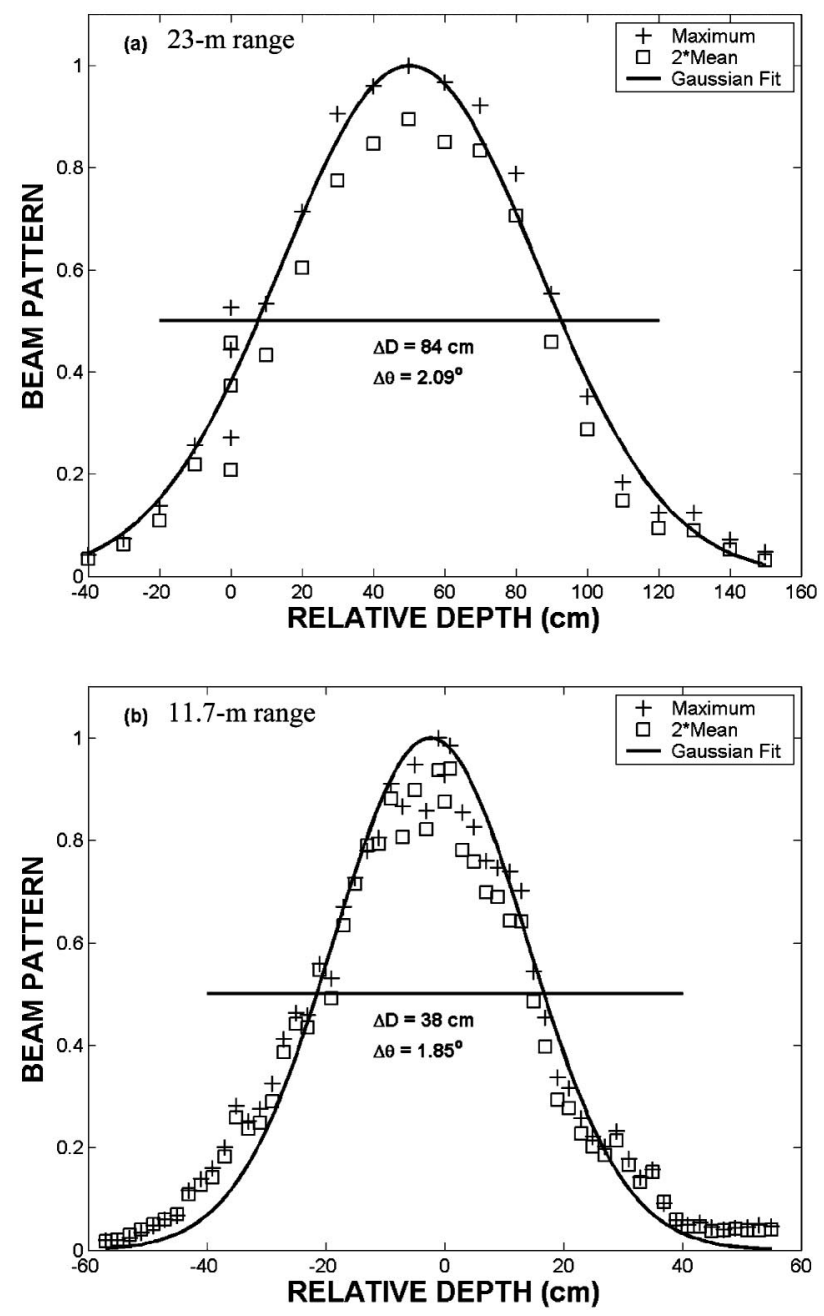

FIG. 5. Directionality of the SM2000/90-kHz multibeam echo sounder in the vertical plane, as determined by changing the depth of the rubber-walled focusing sphere (a) at 23-m range in 4-cm increments in the sea well and (b) at 11.7-m range in 2-cm increments in the freshwater tank. The directionality is expressed through the beam pattern in normalized intensity units. The maximum intensity value is that observed in a spatial window consisting of five contiguous beams and five range cells centered on the respective beam and range of nominal greatest sensitivity. The mean is that of all echo values within the window. A Gaussian function has been fitted to the maximum intensity values.

ing convenient and useful protocols, the transmit power of each sonar was set at that level most often used in field applications.

Measurement of the system response is performed by observing the change in echo amplitude due to changing the receiver gain function of the sonar. It is effected conveniently by observing the change in echo amplitude from a standard target under systematic changes in the receiver gain function. This is illustrated for the SM2000/90-kHz multibeam echo sounder in Fig. 6, where the full range of gain settings has been exercised. A linear function has been fit to the echointensity data by means of a least-squares-regression analysis. ${ }^{45}$ On the basis of the data and their analysis, the gain function $20 \log r+2 \alpha r$ was chosen, where $r$ is the range and $\alpha=0.0157 \mathrm{~dB} / \mathrm{m}$. In the case of the SM2000/90-kHz multibeam echo sounder, the system sensitivity was expressed through a numerical value. This was determined from the equation 


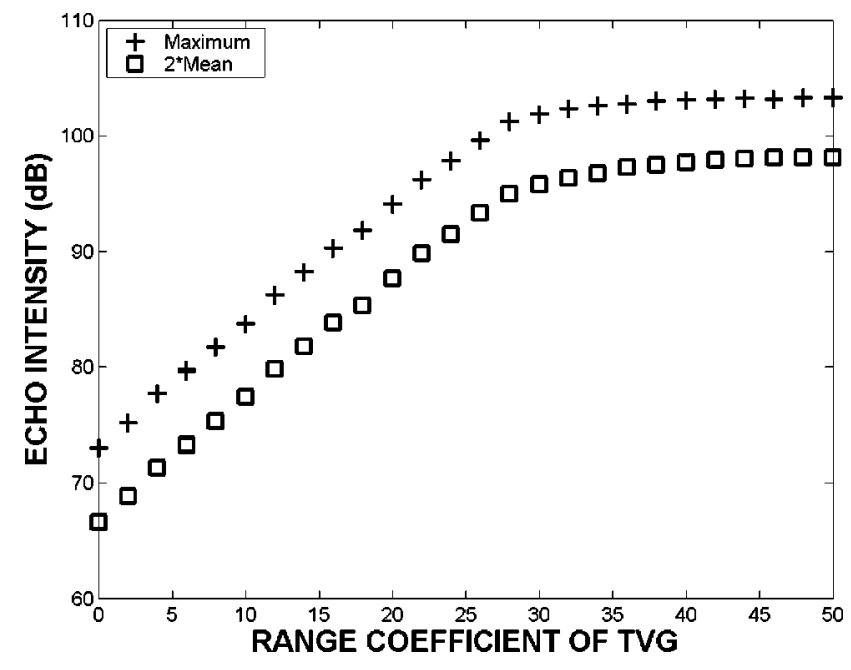

FIG. 6. Measured dependence of the echo intensity from the standard target AL60 on the gain function of the SM2000/90-kHz multibeam echo sounder. The target was measured at $11.7-\mathrm{m}$ range in the sea well, with pulse duration of $300 \mu$ s. For an explanation of maximum and mean values, see the caption to Fig. 5 .

$$
\mathrm{TS}=10 \log _{10}\left(G\left(r_{0}\right)\left|V_{r e c}\right|\right)+20 \log _{10}\left(r_{0}\right)+2 \alpha r_{0},
$$

where $\mathrm{TS}=-34.40 \mathrm{~dB}$ denotes the target strength of the 60 mm-diam aluminum sphere (Sec. II B) at $90 \mathrm{kHz}$ for the particular hydrographic conditions, $r_{0}=11.7 \mathrm{~m}, V_{\text {rec }}$ is a relative digitized real number that is proportional to the received voltage level, and $\alpha=0.0157 \mathrm{~dB} / \mathrm{m}$. It is emphasized that $V_{\text {rec }}$ also depends on the instrument settings, including transmit power level, pulse duration, sampling rate, and assumed absorption coefficient value, which in the present case was $0.0068 \mathrm{~dB} / \mathrm{m}$. Substituting for $V_{\text {rec }}$ and solving, the sensitivity coefficient is $G\left(r_{0}\right)=3.68 \times 10^{-6}$. Multiplying the raw echo amplitude due to an arbitrary target or targets at the same 11.7-m range, with the same gain function, yields backscattering units of square meters. For other gain functions at the same range, the relationship defined in Fig. 6 may be used. Since the calibration was performed in the transducer nearfield, Eq. (1) must be modified for use at other ranges.

\section{Measurement of system stability}

There is little reason to suspect instabilities in performance of multibeam sonars designed for scientific use, although their possible occurrence at high pulse repetition frequencies might be entertained. Ultimately, it is only measurement that can decide the matter or quantify the degree of stability.

Measurement of the system stability is straightforward. The target is held at a fixed position in the transducer beam, and echo measurements are performed over a relatively long period of time. This might last from minutes to hours or days or weeks, the longer period corresponding to potential use on fisheries survey cruises.

An example of a measurement of system stability is presented in Fig. 7. This shows the time series of echo amplitude from the SM2000/200-kHz multibeam echo sounder due to repeated insonification of the standard target WC38.1.

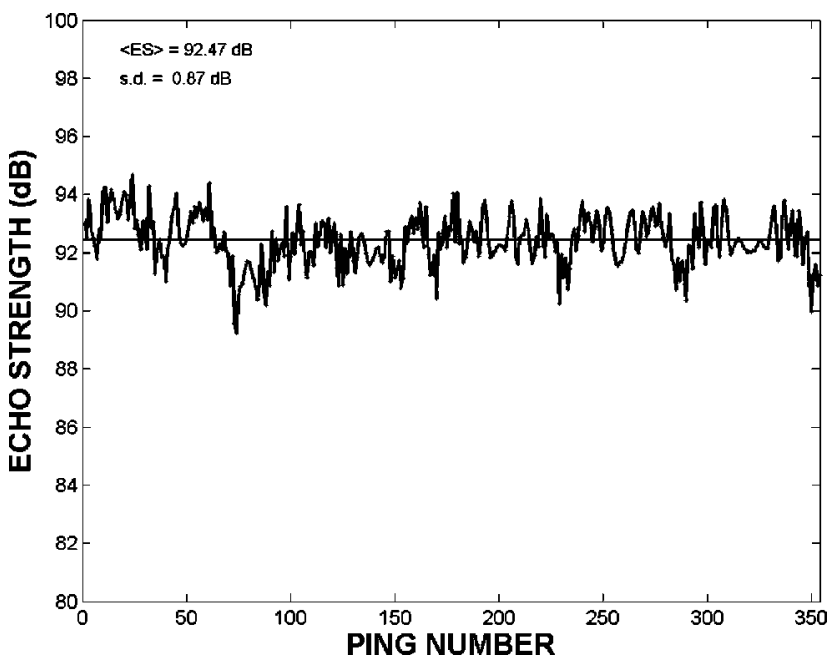

FIG. 7. Echo amplitudes from an on-axis standard target, WC38.1, as measured at $11.7-\mathrm{m}$ range in the freshwater tank by the SM2000/200-kHz multibeam echo sounder over a time period of $175 \mathrm{~s}$.

\section{Measurement of equatorial-plane sensitivity}

For conventional imaging purposes, the equatorial-plane sensitivity function is very important. The value of this function for a small target at a given angle is the peak echo amplitude from among all beams where the target is detected. It is advantageous in imaging applications that the function be relatively flat over the full angular span of the sonar.

The equatorial-plane sensitivity function must vary over the angular span, for it is defined by a finite series of beams of fixed orientation. Adjacent beams meet at a level that depends on the number of beams, their total angular span, the respective aperture size, and the shading of individual elements. For the 128 displayed beams of the SM2000, this level is roughly $2 \mathrm{~dB}$ less than peak levels, thus defining a measure of ripple.

For scientific imaging purposes, knowing the sensitivity function and how it varies is important, for it enables compensation to be effected and distortions removed.

The equatorial-plane sensitivity function can be determined by measuring the echo response of the sonar at closely separated, discrete angles over the full angular span. This was done for the SM2000 multibeam echo sounders and the RESON SeaBat 8101 multibeam sonar. Exemplary resulting functions are shown in Fig. 8.

\section{E. Measurement of central-region directionality in two dimensions}

As mentioned in the previous section, there is particular interest in the equatorial-plane sensitivity function. This is a one-dimensional slice of the more general two-dimensional sensitivity function. Implicit in this description is a rather broad width of the beam in the transverse direction, nominally $20 \mathrm{deg}$ in the vertical plane for the imaging mode of the SM2000. In the echo-sounding mode, the nominal beamwidth is $1.5 \mathrm{deg}$ in the vertical plane.

Again, there is no substitute for measurement of the directionality. This has been done in two dimensions by repeat- 

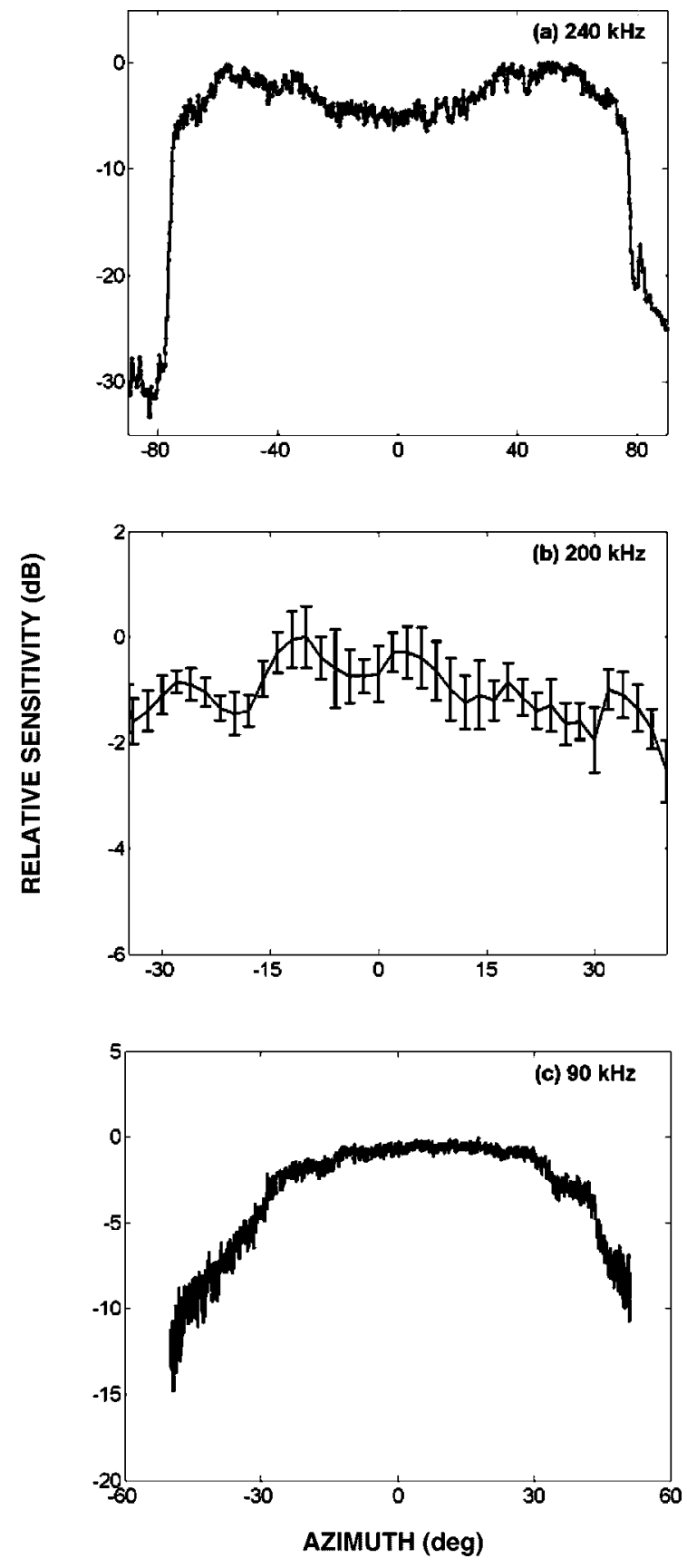

FIG. 8. Measured equatorial-plane sensitivity function of the (a) Seabat $8101240-\mathrm{kHz}$ multibeam sonar, (b) SM2000/200-kHz multibeam echo sounder, and (c) SM2000/90-kHz multibeam echo sounder. The target range was $11.7 \mathrm{~m}$. In (b), only part of the central section of greatest sensitivity was measured.

ing the one-dimensional measurements, with the standard target moved systematically through a series of discrete vertical positions.

Exemplary results of this measurement for the SM2000/ 90-kHz multibeam echo sounder are shown in Fig. 9. Measurements are shown of the central region of directionality.

\section{F. Measurement of directionality of individual beams}

An envisioned major use of multibeam sonar in fisheries survey work is quantification of fish density. For measurements of resolved single fish by echo counting, this requires
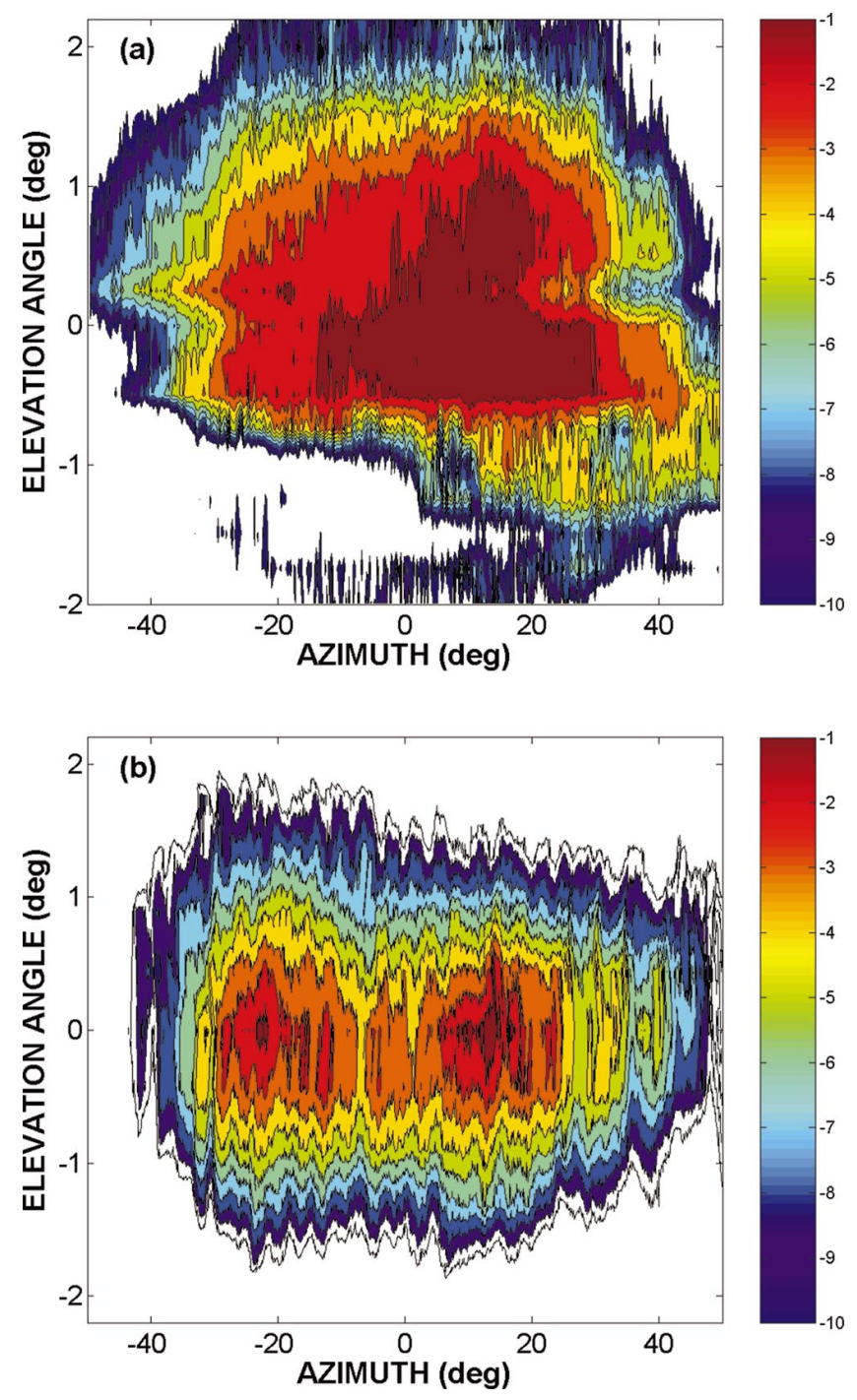

FIG. 9. Measured central-region directionality in two dimensions of the SM2000/90-kHz multibeam echo sounder at (a) 23-m nominal range in the sea well and (b) 11.7-m nominal range in the freshwater tank. The dark brown color represents echo levels extending from -1 to $0 \mathrm{~dB}$.

knowledge of the acoustic sampling volume, ${ }^{46}$ which depends on the individual beam patterns, i.e., the beam patterns of individual apertures used in the quantification. For measurements of unresolved fish aggregations by means of echo integration, ${ }^{1}$ measurement of the area backscattering coefficient requires knowledge of the equivalent beam angle. This is the integral of the product of transmit and receive beam patterns, each expressed in the intensity domain, over all angles. ${ }^{17}$ For directional beams, this angular measure can be determined with high accuracy from the characteristics of the main lobe alone.

Data collected in the measurement of the central-region directionality in two dimensions, described in the previous section, are sufficient to determine this function. Individualaperture beam patterns can also be derived by approximation from measurement of the equatorial-plane sensitivity function, and assumption that the beamwidth in the vertical plane is that measured in the central polar beam pattern ( $\mathrm{Sec}$. IV A). In either case, the individual beam patterns are formed 


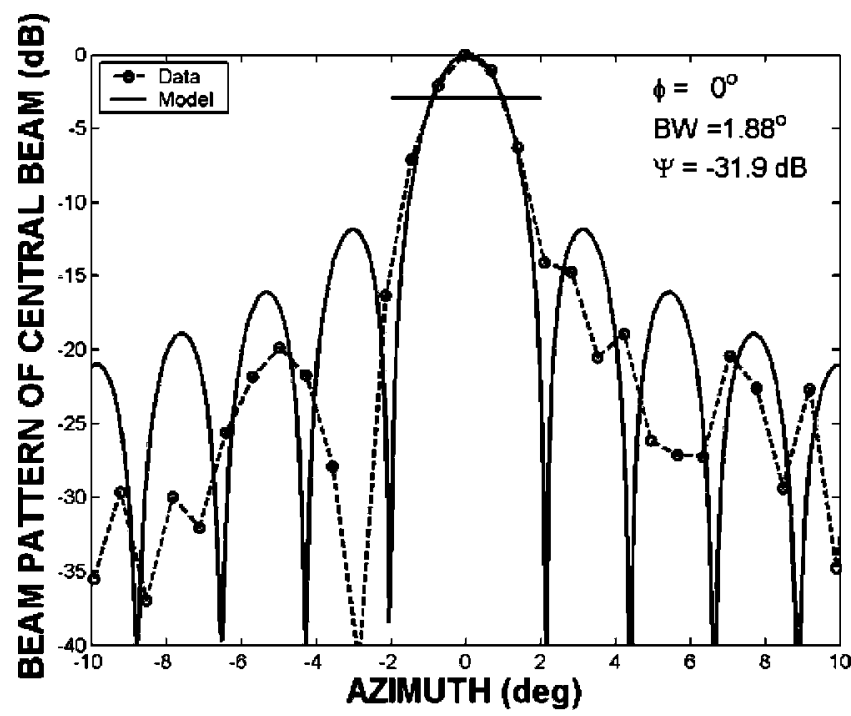

FIG. 10. Equatorial-plane beam patterns, measured and estimated, of a single aperture of the SM2000/90-kHz multibeam echo sounder. The measured value of the beamwidth BW and the estimated value of the equivalent beam angle $\Psi$ are attached to the respective function.

without further selection or reduction, as in the operation of selecting peak values.

Results from the second procedure are shown in Fig. 10 for a single aperture and in Fig. 11 for a set of apertures. Individual beam patterns are shown in the horizontal, equatorial plane together with fitted functions. The inferred equivalent beam angle for the central beam in Fig. 10 is 7.76 $10^{-4} \mathrm{sr}$ or, logarithmically, $-31.1 \mathrm{~dB}$. The theoretical prediction based solely on the geometry of the transducer array is $6.4610^{-4} \mathrm{sr}$ or $-31.9 \mathrm{~dB}$.
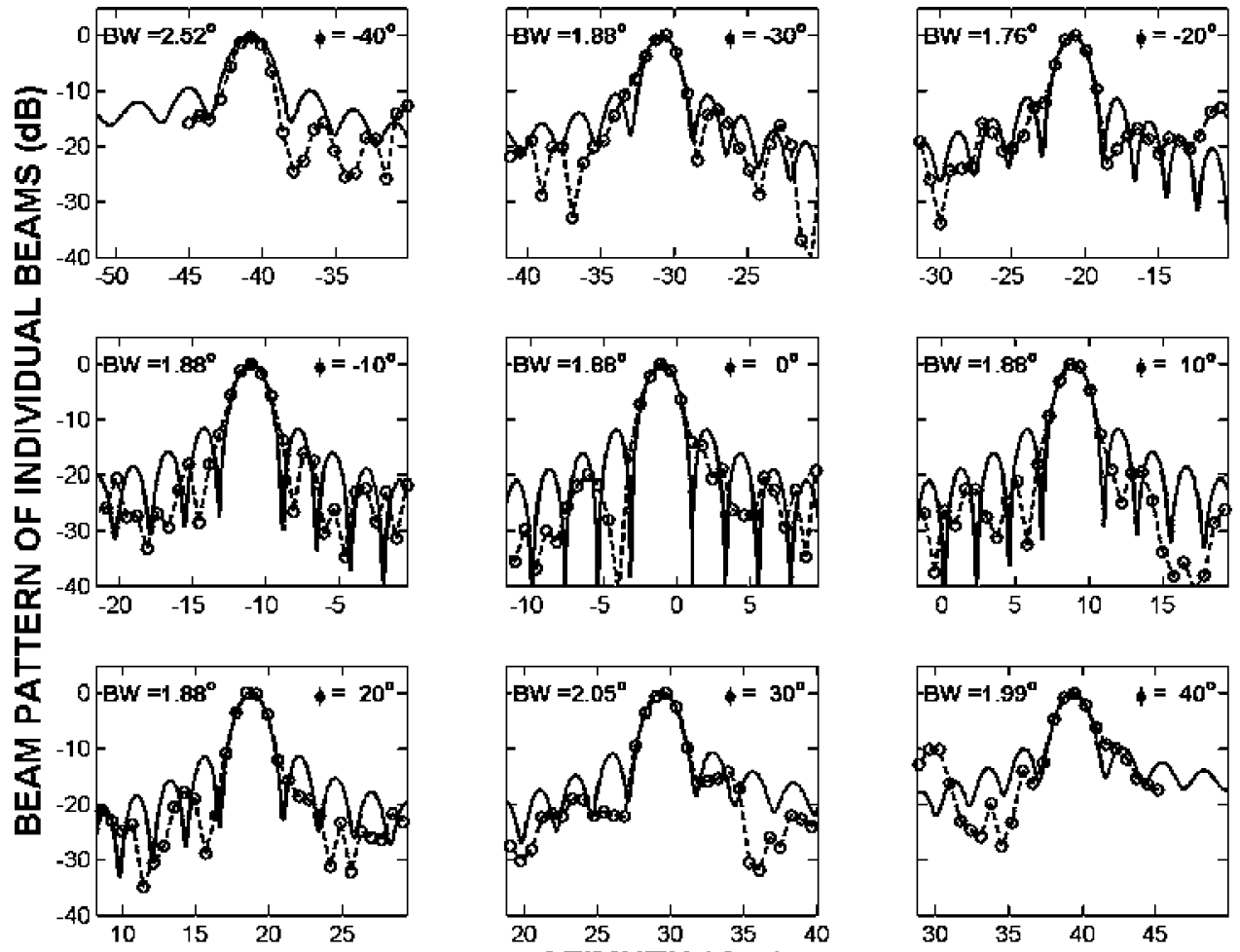

FIG. 11. Equatorial-plane beam patterns, measured and estimated, of a set of individual apertures of the SM2000/ $90-\mathrm{kHz}$ multibeam echo sounder. Measured values of the beamwidth BW are shown. 


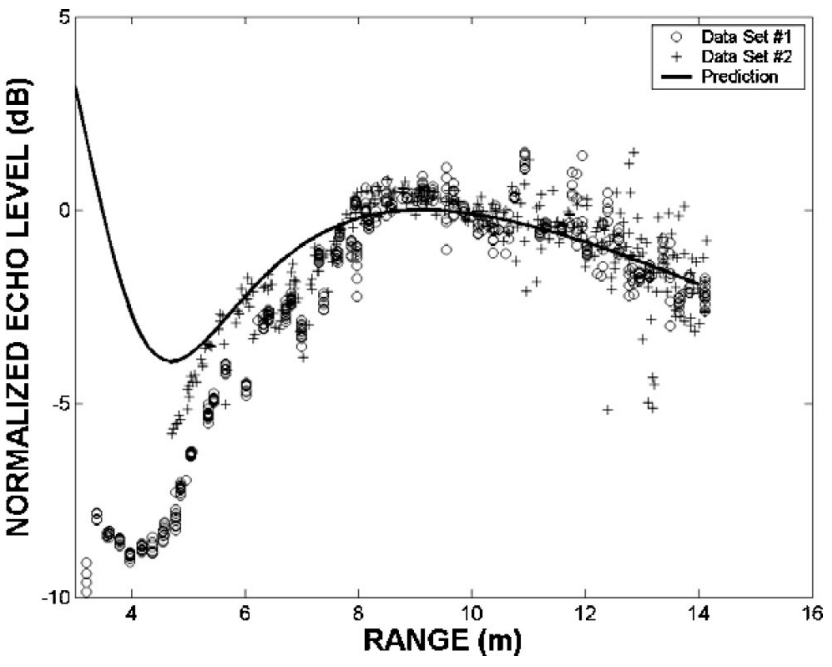

FIG. 12. Dependence of the axial response of the SM2000/90-kHz multibeam echo sounder on range in the transducer nearfield, as measured in the freshwater tank. Superimposed on the data is the response as modeled from the transducer geometry.

carried out in advance of the acoustic measurements. Immediately following a review of the acoustic target-positioning operation, which also accomplishes mapping of the central beam pattern, measurement of the system response and stability, and choice of the receiver gain function, are described. Measurement of system directionality in each of several dimensions is then addressed. Measurement of the noise environment and system gain are also reviewed.

\section{A. Preparations}

In the case of multibeam sonars consisting of separate transmitting and receiving arrays, it may be necessary or advantageous to orient the arrays so that their acoustic axes intersect at the target range. This is particularly important for reducing effects of parallax in the case of nearfield calibration measurements to be applied in the transducer farfield. An easy method for adjusting the relative array orientations is to place shims between the bolts and mounting plate at the far end of one of the transducer arrays.

Another, essential operation in preparing for a calibration exercise is wetting the transducer faces and target to preclude formation of air bubbles on the respective surfaces. ${ }^{47}$ This can be done by washing with a surfactant, for example, a simple household liquid detergent.

Recording the hydrographic state over the range of depths spanned by the transducer and target is essential in any calibration exercise. This is important for excluding stratification and other inhomogeneities in the water column as a cause of refractive effects during a calibration. At sufficiently near ranges, the effect of these on the propagation path between transducer and target may be entirely negligible, but they may be a limiting factor in others. In field applications over longer ranges, especially with horizontal or near-horizontal orientations, refractive effects, which are determined by the hydrography, may be substantial.

Beamforming is essential for the rapid and timely analysis of data. If desired or necessary, it may be performed digitally by software on the basis of the full set of individual element echo signals and detailed knowledge of the receiving aperture geometry. Alternatively, the output signals from the sonar beamformer may be recorded and used directly in the data analyses.

\section{B. Target positioning and measurement of central polar beam pattern}

The basic procedure for relative positioning of the target in the transducer beam has already been outlined in Sec. IV A. It is reduced to a prescription in the following, assuming that the echo measurements are made in or close to the horizontal plane.

The standard target is suspended at a fixed distance from the centered transducer at the nominal transducer depth, specifically, the depth of the center of the transducer array or arrays. The target echo is registered. The target is now lowered by a fixed increment corresponding to some fraction of the nominal beamwidth of the transducer, say one-tenth. The echo measurement at this new position is repeated. The target is lowered again by the same fixed increment and its echo is recorded. This process is repeated until the first null is passed, which can be determined by observing a rise in the echo amplitude immediately following a steady decrease. The target is returned to the first measurement depth and the original measurement is repeated. The target is now raised by the fixed increment, and the echo is recorded. This process is repeated until the first null is again passed.

Beamforming is performed, if necessary, and the peak amplitude from the full set of beams is selected and plotted. The central equatorial plane of sensitivity is defined generally by the maximum in the plotted function. This may be determined by simple visual inspection if unambiguous, otherwise by fitting a quadratic or other nonlinear function to the data.

The exact position of the central axis can now be determined by rotating the transducer and observing the peak echo in the vicinity of the mechanically determined central axis. If the signal-to-noise ratio is sufficiently high, phase differences can be used to determine the angle of greatest sensitivity, which defines the central axis. ${ }^{48}$ In either case, this refined determination of the central axis can reveal possible mechanical or electrical offsets in the central acoustic axis of the sonar. If the central axis deviates very much from the assumed axis, the measurements of polar directionality can be repeated to ensure their precision.

In the course of finding the central equatorial plane, the central polar beam pattern is measured. Noncentral polar beam patterns can be determined in a similar way.

\section{System response, stability, and choosing receiver gain function}

Having found the central plane of sensitivity, the system response can be determined for particular array orientations by observing the echo. By relating the measured output, e.g., amplitude, to the corresponding input signal and backscattering properties of the standard target, the transfer function of the system can be measured. This may involve analyzing the 
frequency content of the transmitted signal and frequency dependence of the target backscattering cross section.

By repeating the measurements for the same transducer orientation and target position over a period of time, the stability of the system can be gauged. This can be quantified by a conventional time-series analysis, with observation of possible changes in the mean and variance over time.

By repeating the measurements with different receiver gain functions, the dependence of the system response on this function can be determined. By fitting a linear function to the echo intensity data, a suitable time-varied-gain function for the measurements can be determined. The system performance at the selected gain function can be related to other gain functions by means of the repeated measurements of system response.

\section{Equatorial-plane sensitivity}

Given knowledge of the location of the central plane of sensitivity, the equatorial-plane sensitivity function can be determined by rotating the transducer array or arrays over the angular span of interest. At each individual orientation, the several beam outputs are examined and the peak amplitude is selected. This process is repeated over the full angular span. The function defined by the peak output versus angle constitutes the equatorial-plane sensitivity.

\section{E. Central-region directionality in two dimensions}

The measurements of the equatorial-plane sensitivity function are now repeated at each of a succession of target depths. As in the target-positioning operation (Sec. V B), the target is moved systematically from the central plane past the first null below the plane, then from the central plane past the first null above the plane. Knowing the full target depth range spanning the main lobe, the measurements can also be performed in a single direction from one null to the other. By measuring the sensitivity function at each of these discrete target depths, the central-region directionality can be mapped in two dimensions.

\section{F. Directionality of individual beams}

The directionality of individual beams is measured in the central plane of sensitivity, as in Sec. VD, and over the central region of sensitivity, as in Sec. VE. The basic beamformed outputs are identical. Instead of selecting peak amplitudes from the range of beams for each transducer orientation, the amplitudes are associated with the respective individual beam. The individual beam patterns can thus be mapped. Individual beams are conveniently identified by their order in the series or by their nominal angle of peak sensitivity.

\section{G. Nearfield response along acoustic axis}

In cases where the multibeam sonar is to be used in the transducer nearfield or where the sonar is to be used in the farfield but can only be calibrated in the nearfield, the nearfield response should be measured. This is done by moving either the transducer or standard target along the acoustic axis and measuring the echo response at discrete positions. The beamformed results are plotted against the transducertarget range.

The measurements are used for comparison against computations based on the transducer geometry and transmit frequency. Confirmation of the computational results provides support for the model, which can then be used to extrapolate either to greater or lesser ranges, depending on the intended sonar application.

\section{H. Noise measurements}

In all of the foregoing protocols, the echo from a standard target was generally measured. These measurements should be accompanied by similar measurements made in the absence of the target, hence of volume reverberation.

The reverberation measurements might be performed conveniently immediately before or immediately after a series of measurements with a standard target. While echoes from transient targets such as schools of juvenile fish passing through the sea well might not be recorded, the general level of background reverberation would be. Such measurements are important for describing the reverberation background and for quantifying the signal-to-noise ratio present during the target measurements. If the reverberation level is sufficiently high, another target with greater target strength might be chosen to ensure a better signal-to-noise ratio.

\section{Electronic measurements}

While purely electronic measurements are inadequate for determining the overall transmit-receive sensitivity, because of neglect of the transducer, they are nonetheless very useful for determining how signals are transferred in the receiver. The basis of the measurements is that of the timeamplitude-frequency measurement method. ${ }^{49}$

The transducer is decoupled from the receiver. Signals of known amplitude and time delay are introduced into each channel, and the output is observed over a range of amplitudes sufficient to test the dynamic range and application of gain to each signal. The measurement sequence is repeated for each transducer element.

A complication with this simple notion is that of multiplexing of the individual transducer element signals before transmission to the beamforming processor, i.e., beamformer. Introduction of a signal of known amplitude and time delay is thus made to a multiplexer, with reception at the end of a cable. A special manufacturer-built unit can be entertained for measurement of the overall receiver sensitivity function with decoupled transducer. Such a capability would allow measurement of the electronic system to be undertaken independently of that of the combined acoustic and electronic system.

\section{DISCUSSION}

By means of the calibration protocols described here, which have been developed through actual testing in nearly every instance, the performance of multibeam sonars can be defined. The general system sensitivity can be expressed through transfer characteristics and measures of directional- 
ity. In addition, the stability can be quantified, and the connection between nearfield and farfield characteristics can be established.

The standard-target calibration method, which has been extended to multibeam sonar, is useful for its absolute character. Multibeam sonars calibrated in this way can be used in quantitative applications, for example, to estimate the numerical density of fish aggregations through echo-counting or echo-integration techniques. Visualization of fish aggregations by multibeam sonar, which is more often accomplished by uncalibrated systems, also benefits from a standard-target calibration, for variations in angular sensitivity can be compensated, giving a more accurate impression of the relative concentration densities.

Another practical advantage of calibration is making possible intercomparability of measurements with different multibeam sonars. Whether the several systems operate at the same or different frequencies, the measurements can be performed in an absolute sense. Given knowledge of the backscattering properties of the target, including the frequency dependence of target strength, if necessary the several acoustic measurements can be converted to biological or other physical measures of density, opening the possibility of direct comparison.

While the present work is directed to the calibration of multibeam sonars used for water-column measurements, it can also be applied to bathymetric multibeam sonars. In addition to measuring the range to the seafloor over a swath, the backscattering properties of the same can be measured and expressed in absolute units of surface scattering. Such measurements are essential for characterizing the seafloor and detecting changes in this, for example, to observe possible changes to the seafloor over time, as due to natural or man-made disturbances.

By quantifying the performance of multibeam sonar, conformity of manufactured units to their specifications can be confirmed. In addition, the performances of different sonars can be compared.

A number of outstanding problems in multibeam sonar calibration are identified. One is to generalize Eq. (1) to arbitrary operating conditions and ranges spanning the nearfield-farfield transition region of the sonar transducer. Another is to identify the causes of variability, for example, those encountered in measurements of system stability, already noted in Sec. IV C and observed in Fig. 8, as well as in Ref. 50. A third problem is to realize the electronic measurements described in Sec. VI in a rapid, automated manner. This could be done through a computer-controlled timeamplitude-frequency device. Another problem is extending the calibration protocols to multibeam sonars as configured for use, for example, as mounted within a sonar dome on the hull of a research vessel or on a fixed structure.

It is appreciated that rigorous calibration of multibeam sonars as configured for use may be difficult. In such cases, confirmation of the performance rather than measurement of the performance can be entertained. The problem of validation is thus identified. Derivative procedures, possibly with relaxed tolerances, could be very useful.

\section{SUMMARY}

The goal of the project documented here was to develop convenient protocols for the calibration of multibeam sonar. The protocols that have been developed, based on the standard-target method, will enable absolute physical measurements to be made with the sonar. Addressing identified outstanding issues will enable the protocols to be extended efficiently to sonars as configured for use. It will also enable the overall accuracy of echo signal processing in the receiver to be quantified.

\section{ACKNOWLEDGMENTS}

The following are thanked for their participation in one or more of the calibration trials: B. Doherty, K. W. Doherty, S. P. Liberatore, and M. Parmenter, Woods Hole Oceanographic Institution; P. Lavoie, A. McLeod, and J. Millar, University of New Hampshire; Y. Li, formerly at the University of New Brunswick, Canada; G. D. Melvin, Department of Fish and Oceans, Canada; W. Michaels and D. A. Perry, Northeast Fisheries Science Center, Woods Hole. A. Bowen and D. R. Yoerger, Woods Hole Oceanographic Institution, are thanked for allowing their 6000-m-depth-rated SM2000/ $200-\mathrm{kHz}$ multibeam imaging sonar to be used in an early trial. Several multibeam sonar manufacturers and their representatives are thanked for lending or arranging loans of sonars for use in developing the calibration protocols, as well as for participating in various trials. These include R. Asplin and C. Smith, Kongsberg Simrad Mesotech, Port Coquitlam, B.C., Canada; J. Condiotty, Simrad, Inc.; and S. Johnson and R. Morton, RESON, Inc. L. Bjørnø, J. Steensrup, and P. K. Eriksen are thanked for providing data on the RESON SeaBat 8101 sonar array. J. Doucette is thanked for assistance in preparing figures; S. Barkley, A. Norton, and M. Parmenter are thanked for assistance in assembling the manuscript. Support by the National Science Foundation through Award No. OCE-0002664, NOAA through Grant No. NA97OG0241, and the Cooperative Institute for Climate and Ocean Research (CICOR) through NOAA Contract No. NA17RJ1223 is acknowledged. This is Woods Hole Oceanographic Institution Contribution No. 11178.

${ }^{1}$ H. Medwin and C. S. Clay, Fundamentals of Acoustical Oceanography (Academic, Boston, 1998).

${ }^{2}$ R. B. Mitson, Fisheries Sonar (Fishing News Books, Farnham, Surrey, England, 1983).

${ }^{3}$ O. A. Misund and A. Aglen, "Swimming behavior of fish schools in the North Sea during acoustic surveying and pelagic trawl sampling," ICES J. Mar. Sci. 49, 325-334 (1992).

${ }^{4}$ F. Gerlotto, P. Fréon, M. Soria, P. H. Cottais, and L. Ronzier, "Exhaustive observations of the 3D schools structure using multibeam sidescan sonar: potential use for school classification, biomass estimation, and behavior studies," Council Meeting of the Int. Council Explor. Sea 1994/B:26 (1994).

${ }^{5}$ F. Gerlotto, M. Soria, and P. Fréon, "From two dimensions to three: the use of multibeam sonar for a new approach in fisheries acoustics," Can. J. Fish. Aquat. Sci. 56, 6-12 (1999).

${ }^{6}$ M. Soria, P. Fréon, and F. Gerlotto, "Analysis of vessel influence on spatial behaviour of fish schools using a multi-beam sonar and consequences for biomass estimates by echo-sounder," ICES J. Mar. Sci. 53, 453-458 (1996).

${ }^{7}$ O. A. Misund, "Dynamics of moving masses: variability in packing den- 
sity, shape, and size among herring, sprat and saithe schools," ICES J. Mar. Sci. 50, 145-160 (1993).

${ }^{8}$ M. T. Hafsteinsson and O. A. Misund, "Recording the migration behavior of fish schools by multibeam sonar during conventional acoustic surveys," ICES J. Mar. Sci. 52, 915-924 (1995).

${ }^{9}$ D. G. Reid (ed.), "Report on echo trace classification," ICES Coop. Res. Rep. (238) (2000).

${ }^{10}$ L. Mayer, Y. Li, and G. Melvin, "3D visualization for pelagic fisheries research and assessment," ICES J. Mar. Sci. 59, 216-225 (2002).

${ }^{11}$ T. C. Gallaudet and C. P. de Moustier, "High-frequency volume and boundary acoustic backscatter fluctuations in shallow water," J. Acoust. Soc. Am. 114, 707-725 (2003).

${ }^{12}$ L. Nøttestad and B. E. Axelsen, "Herring schooling manoeuvres in response to killer whale attacks," Can. J. Zool. 77, 1540-1546 (1999).

${ }^{13}$ B. E. Axelsen, T. Anker-Nilssen, P. Fossum, C. Kvamme, and L. Nøttestad, "Pretty patterns but a simple strategy: predator-prey interactions between juvenile herring and Atlantic puffins observed with multibeam sonar," Can. J. Zool. 79, 1586-1596 (2001).

${ }^{14} \mathrm{~K}$. Benoit-Bird and W. Au, "Hawaiian spinner dolphins aggregate midwater food resources through cooperative foraging," J. Acoust. Soc. Am. 114, 2300 (2003)

${ }^{15}$ T. Weber, D. Bradley, R. L. Culver, and A. Lyons, "Inferring the vertical turbulent diffusion coefficient from backscatter measurements with a multibeam sonar," J. Acoust. Soc. Am. 114, 2300 (2003).

${ }^{16}$ C. D. Jones, "Water-column measurements of hydrothermal vent flow and particulate concentration using multibeam sonar," J. Acoust. Soc. Am. 114, 2300-2301 (2003).

${ }^{17}$ R. J. Urick, Principles of Underwater Sound, 3rd ed. (McGraw-Hill, New York, 1983)

${ }^{18}$ K. G. Foote, "Optimizing copper spheres for precision calibration of hydroacoustic equipment," J. Acoust. Soc. Am. 71, 742-747 (1982).

${ }^{19}$ K. G. Foote, "Maintaining precision calibrations with optimal copper spheres," J. Acoust. Soc. Am. 73, 1054-1063 (1983).

${ }^{20}$ K. G. Foote, H. P. Knudsen, G. Vestnes, D. N. MacLennan, and E. J. Simmonds, "Calibration of acoustic instruments for fish density estimation: a practical guide," ICES Coop. Res. Rep. 144 (1987).

${ }^{21}$ D. Chu, K. G. Foote, and L. C. Hufnagle, Jr., "Measurement of multibeam sonar directivity patterns," Proc. MTS/IEEE Oceans 2002 Conf., pp. 1411-1414 (2002)

${ }^{22}$ N. A. Cochrane, Y. Li, and G. D. Melvin, "Quantification of a multibeam sonar for fisheries assessment applications," J. Acoust. Soc. Am. 114, 745-758 (2003).

${ }^{23}$ D. Chu, K. G. Foote, L. C. Hufnagle, Jr., K. C. Baldwin, L. A. Mayer, and A. McLeod, "Calibrating a 90-kHz multibeam sonar," Proc. MTS/IEEE Oceans 2003 Conf., pp. 1633-1636 (2003).

${ }^{24}$ K. G. Foote, D. Chu, K. C. Baldwin, L. A. Mayer, A. McLeod, L. C. Hufnagle, Jr., J. M. Jech, and W. Michaels, "Protocols for calibrating multibeam sonar," J. Acoust. Soc. Am. 114, 2307 (2003).

${ }^{25}$ G. C. Gaunaurd, "Sonar cross section of bodies partially insonified by finite sound beams," IEEE J. Ocean. Eng. OE-10, 213-230 (1985).

${ }^{26}$ A. D. Poularikas, "Fourier transforms and the fast Fourier transform," in The Electrical Engineering Handbook, 2nd edition, edited by R. C. Dorf (CRC, Boca Raton, FL, 1997), pp. 287-298.

${ }^{27}$ K. G. Foote and D. N. MacLennan, "Comparison of copper and tungsten carbide calibration spheres," J. Acoust. Soc. Am. 75, 612-616 (1984)

${ }^{28}$ K. G. Foote, P. R. Atkins, C. C. Bongiovanni, D. T. I. Francis, P. K.
Eriksen, M. Larsen, and T. Mortensen, "Measuring the frequency response function of a seven-octave-bandwidth echo sounder," Proc. Inst. Acoust. 21(1), 88-95 (1999).

${ }^{29}$ K. G. Foote, D. T. I. Francis, H. Furset, and H. Hobæk, "Spheres for calibrating high-frequency broadband echo sounders," Acust. Acta Acust. 85, S186-S187 and J. Acoust. Soc. Am. 105, 1112-1113(A) (1999).

${ }^{30}$ D. L. Folds, "Target strength of focused liquid-filled spherical reflectors," J. Acoust. Soc. Am. 49, 1596-1599 (1971)

${ }^{31}$ R. H. Wallace, H. V. Hillery, G. R. Barnard, B. M. Marks, and C. M. McKinney, "Experimental investigation of several passive sonar targets," J. Acoust. Soc. Am. 57, 862-869 (1975).

${ }^{32}$ B. M. Marks and E. E. Mikeska, "Reflections from focused liquid-filled spherical reflectors,” J. Acoust. Soc. Am. 59, 813-817 (1976).

${ }^{33}$ D. L. Folds and C. D. Loggins, "Target strength of liquid-filled spheres," J. Acoust. Soc. Am. 73, 1147-1151 (1983)

${ }^{34}$ T. R. Hammar, K. G. Foote, and S. P. Liberatore, "Advances in developing a high-frequency sonar calibration facility," Proc. MTS/IEEE Oceans 2003 Conf., pp. 1622-1624 (2003).

${ }^{35}$ D. M. Deveau, "Fabrication, testing, and installation of fluid-filled target spheres for AUTEC's passive sonar calibration system," Proc. MTS/IEEE Oceans 2000 Conf., pp. 1481-1487 (2000).

${ }^{36}$ J. J. Faran, Jr., "Sound scattering by solid cylinders and spheres," J. Acoust. Soc. Am. 23, 405-418 (1951)

${ }^{37}$ R. Hickling, "Analysis of echoes from a solid elastic sphere in water," J. Acoust. Soc. Am. 34, 1582-1592 (1962)

${ }^{38}$ R. R. Goodman and R. Stern, "Reflection and transmission of sound by elastic spherical shells," J. Acoust. Soc. Am. 34, 338-344 (1962).

${ }^{39}$ K. W. Doherty, T. R. Hammar, and K. G. Foote, "Transducer mounting and rotating system for calibrating sonars in a sea well," Proc. MTS/IEEE Oceans 2002 Conf., pp. 1407-1410 (2002).

${ }^{40}$ K. C. Baldwin, L. Mayer, A. McLeod, and J. Millar, "Acoustic transducer calibration system,” Proc. MTS/IEEE Oceans 2003 Conf., pp. 2093-2099 (2003).

${ }^{41}$ R. F. Duncan and F. S. Blanchard, A Cruising Guide to the New England Coast, 5th ed. (Dodd, Mead, New York, 1962).

${ }^{42}$ C. W. Horton, Sr., Signal Processing of Underwater Acoustic Waves (U.S. Government Printing Office, Washington, DC, 1969).

${ }^{43}$ W. B. Davenport, Jr., "Signal-to-noise ratios in band-pass limiters," J. Appl. Phys. 24, 720-727 (1953).

${ }^{44}$ H. Bodholt, H. Nes, and H. Solli, "A new echo-sounder system," Proc. Inst. Acoust. 11(3), 123-130 (1989).

${ }^{45}$ N. R. Draper and H. Smith, Applied Regression Analysis (Wiley, New York, 1966)

${ }^{46}$ K. G. Foote, “Acoustic sampling volume," J. Acoust. Soc. Am. 90, 959964 (1991).

${ }^{47}$ L. E. Ivey and C. M. Thompson, "Underwater transducer wetting agents," J. Acoust. Soc. Am. 78, 389-394 (1985).

${ }^{48}$ D. Chu, K. C. Baldwin, K. G. Foote, Y. Li, L. A. Mayer, and G. D. Melvin, "Multibeam sonar calibration: target localization in azimuth," Proc. MTS/IEEE Oceans 2001 Conf., pp. 2506-2510 (2001).

${ }^{49}$ H. P. Knudsen, "T-A-F: Time-amplitude-frequency: A special electronic unit for measuring the TVG function in research echo-sounders," Fiskeridir. Skr., Ser. Havunders. 17, 529-541 (1985).

${ }^{50}$ D. Chu, K. G. Foote, T. R. Hammar, L. C. Hufnagle, Jr., and J. M. Jech, "Calibrating a 90-kHz multibeam sonar: illustrating protocols," Proc. MTS/IEEE Oceans 2004 Conf., pp. 438-442 (2004) 